\title{
ON INSTABILITY \\ OF THE ABSOLUTELY CONTINUOUS SPECTRUM OF DISSIPATIVE SCHRÖDINGER OPERATORS AND JACOBI MATRICES
}

\author{
R. ROMANOV
}

\begin{abstract}
The absence of the absolutely continuous spectrum is proved for dissipative Schrödinger operators and Jacobi matrices with slowly decaying imaginary part of the potential.
\end{abstract}

\section{§1. INTRODUCTION}

This paper addresses the question of stability of the absolutely continuous spectrum of one-dimensional Schrödinger operators under slowly decaying perturbations. In the selfadjoint case the absolutely continuous spectrum coincides with the spectrum of the unperturbed operator on the positive real axis (see [1]) if the potential is of class $L^{2}$. In particular, this result means that the absolutely continuous spectrum can be preserved when conventional scattering theory is no longer applicable. Here the paper 2 should be mentioned, where a scattering theory was constructed for potentials belonging to $L^{2-\varepsilon}$, $\varepsilon>0$. Our aim is to show that the picture is very different for nonselfadjoint Schrödinger operators. Namely, the following two theorems hold. ${ }^{1}$

Theorem 1. Let $\left\{q_{j}\right\}$ be a sequence of complex numbers with $\operatorname{Im} q_{j} \geq 0$ and $\operatorname{Im} q_{j} \rightarrow 0$, and let $l$ be the discrete Schrödinger operator in $l^{2}(\mathbb{N})$ determined by the matrix

$$
\left(\begin{array}{cccc}
q_{1} & 1 & 0 & \cdots \\
1 & q_{2} & 1 & \ddots \\
0 & 1 & q_{3} & \ddots \\
\vdots & \ddots & \ddots & \ddots
\end{array}\right) .
$$

If the absolutely continuous subspace of $l$ is nontrivial, then $\operatorname{Im} q \in l^{1}$.

Theorem 2. Let $q$ be a locally bounded function on $\mathbb{R}_{+}$with $\operatorname{Im} q \geq 0$ and $\operatorname{Im} q(x) \rightarrow 0$ as $x \rightarrow \infty$. Assuming that $\operatorname{Re} q$ is bounded below and $\sup _{n} \int_{n}^{n+1}|\operatorname{Re} q|^{2} d t$ is finite, define $l$ to be the Schrödinger operator in $L^{2}\left(\mathbb{R}_{+}\right)$with the potential $q$, lu $=-u^{\prime \prime}+q u$, on the domain fixed by a selfadjoint boundary condition at zero. If the absolutely continuous subspace of $l$ is nontrivial, then $\operatorname{Im} q \in L^{1}$.

2000 Mathematics Subject Classification. Primary 34L10, 47B44.

Key words and phrases. Absolutely continuous subspace, dissipative operators, Schrödinger operator. The author was supported in part by EPSRC Grant GR/R20885 and by RFBR (grant no. 00-0100479).

${ }^{1}$ See (2.1) for the definition of the absolutely continuous subspace for the class of operators under consideration. 
The abstract local scattering theory for trace class perturbations of selfadjoint operators, developed in [3, 4, shows that the absolutely continuous parts of arbitrary operators $L, L_{0}$ are quasisimilar and their spectra coincide if $L_{0}$ is selfadjoint and $L-L_{0}$ is of trace class. In the situation under consideration this implies that the absolutely continuous spectrum of $l$ coincides with that of the selfadjoint Schrödinger operator Re $l$ provided $\operatorname{Im} q \in L^{1}\left(\operatorname{Im} q \in l^{1}\right.$ in the discrete case). Thus, the theorems above say that the existence of the absolutely continuous spectrum for Schrödinger operators in the dissipative case is equivalent to having a nontrivial scattering theory. To the best of the author's knowledge, no nontrivial negative results on the absolutely continuous spectrum of nonselfadjoint differential operators with nondiscrete spectrum have been obtained previously.

Notice that we managed to avoid using explicitly the Szökefalvi-Nagy-Foiaş functional model [5], which is the main tool in the study of the absolutely continuous subspace of a dissipative operator [3, 6. However, a model-oriented reader will easily recognize the condition of isometricity a.e. on the real axis of the characteristic function of the operator in question in the basic criterion (2.2).

The structure of the paper is as follows. In $\S 2$ we define the absolutely continuous subspace of a dissipative operator and provide necessary abstract preliminaries. $\S 3$ is devoted to the proof of Theorem $1, \S 4$ to the proof of Theorem 2.

\section{Notation.}

$\mathbb{C}_{ \pm}=\{z: \pm \operatorname{Im} z>0\}$.

$H_{\mathrm{ac}}(L)$ is the absolutely continuous subspace of an operator $L$. The notation $H_{\mathrm{ac}}$ without an argument is used when from the context it is clear which operator it refers to.

$\mathbf{S}^{1}$ is the trace class.

$T(x, k)$ is the transfer matrix for the differential equation $-y^{\prime \prime}+p y=k y$ on the semiaxis $x \geq 0$ :

$$
T(x, k)\left(\begin{array}{c}
y(0) \\
y^{\prime}(0)
\end{array}\right)=\left(\begin{array}{c}
y(x) \\
y^{\prime}(x)
\end{array}\right)
$$

for any solution $y$ of the differential equation.

$\|\cdot\|_{X}=\|\cdot\|_{L^{2}(0, X)} \cdot$

$\sigma_{\text {ess }}(L)$ is the essential spectrum of the operator $L$. We write $\sigma_{\text {ess }}(L) \subset \Omega$ if the spectrum of $L$ in the complement of the set $\Omega$ consists of at most countably many eigenvalues of finite geometric multiplicity.

Unless otherwise specified, the abbreviation a.e. refers to the Lebesgue measure.

\section{§2. Absolutely continuous subspace}

Let $H$ be a Hilbert space, and let $L$ be a maximal dissipative operator in $H$ of the form $L=A+i V$, where $A=A^{*}$ and $V \geq 0$ is bounded. We shall assume that $\sigma_{\text {ess }}(L) \subset \mathbb{R}$. It is also assumed throughout that the operator $L$ is completely nonselfadjoint, that is, has no reducing subspaces on which it induces a selfadjoint operator. We define the Hardy classes of vector-valued functions $\mathbf{H}_{ \pm}^{2}$ to be the collections of functions $f: \mathbb{C}_{ \pm} \rightarrow H$ analytic in $\mathbb{C}_{ \pm}$, respectively, and satisfying $\sup _{\varepsilon>0} \int_{\mathbb{R}}\|f(k \pm i \varepsilon)\|^{2} d k<\infty$.

Definition. The absolutely continuous subspace $H_{\mathrm{ac}}(L)$ of the operator $L$ is defined as follows [3, 7]:

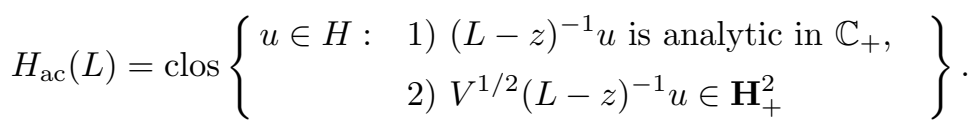


The absolutely continuous subspace is regular invariant 3 ; that is, $\overline{(L-z)^{-1} H_{\mathrm{ac}}}=$ $H_{\mathrm{ac}}$ for all $z \in \rho(L)$. This implies that the restriction $\left.L\right|_{H_{\mathrm{ac}}}$ with the domain $\mathcal{D}(L) \cap H_{\mathrm{ac}}$ is a closed operator in $H_{\mathrm{ac}}$.

Various motivations of this definition and its relationship with scattering theory are discussed in [3, 4, 7, 6, 8,. Here we only mention the following "weak" characterization of the subspace $H_{\mathrm{ac}}(L)$ (it will not be used in the paper).

Theorem (see [10]).

$$
H_{\mathrm{ac}}(L)=\operatorname{clos}\left\{\begin{array}{l}
u \in H: \quad \text { 1) }(L-z)^{-1} u \text { is analytic in } \mathbb{C}_{+}, \\
\text {2) }\left.\left\langle(L-z)^{-1} u, v\right\rangle\right|_{\mathbb{C}_{ \pm}} \in \mathbf{H}_{ \pm}^{2} \text { for all } v \in H
\end{array}\right\} .
$$

This theorem shows, in particular, that (2.1) can be regarded as a generalization of the definition of the absolutely continuous subspace in the selfadjoint theory.

The triviality of the subspace $H_{\mathrm{ac}}$ in the examples considered in this paper will be established on the basis of the following criterion, which is implicit in [8].

Proposition 2.1. $H_{\mathrm{ac}}(L)=\{0\}$ if and only if for a.e. $k \in \mathbb{R}$ we have $(z=k+i \varepsilon)$

$$
D(z) \equiv \sqrt{\varepsilon}\left(L^{*}-z\right)^{-1} \sqrt{V} \stackrel{s}{\longrightarrow} 0
$$

as $\varepsilon \downarrow 0$.

Notice that it suffices to verify strong convergence on a dense set, because the function $D(z)$ is bounded in $\mathbb{C}_{+},\|D(z)\| \leq 1 / 2$ (see a calculation in [8] or the proof of Lemma 2.3 below, where a similar calculation is carried out). To make the exposition self-contained, here we give an elementary proof of the "if" part (the "only if" part is not needed in this paper). In the argument we shall use the following inequality from [3]:

$$
\varepsilon \int_{\mathbb{R}}\left\|\left(L^{*}-k-i \varepsilon\right)^{-1} v\right\|^{2} d k \leq \pi\|v\|^{2}
$$

which is valid for any maximal dissipative operator $L$ and all $v \in H$ and $\varepsilon>0$. This inequality is an easy consequence of the existence of a selfadjoint dilation [5] of the maximal dissipative operator $-L^{*}$; see 3 for the details.

Proof. Given a $u \in H$, we define $u_{ \pm}(z), z \in \mathbb{C}_{ \pm}$, to be the restrictions of the function $\sqrt{V}(L-z)^{-1} u$ to $\mathbb{C}_{ \pm}$, respectively. By a fundamental property of dissipative operators, $u_{-} \in \mathbf{H}_{-}^{2}$ for all $u \in H\left[3\right.$, Theorem 1]. Suppose (2.2) is fulfilled and $u_{+} \in \mathbf{H}_{+}^{2}$ for a certain $u \in H$. We shall show that $u=0$. Define $u_{ \pm}(k) \in L^{2}(\mathbb{R}, H)$ to be the boundary values of the functions $u_{ \pm}$on the real axis. The required assertion will be established if we show that $u_{+}=u_{-}$in $L^{2}(\mathbb{R}, H)$. For all $z=k+i \varepsilon, \varepsilon>0$, and $e \in H$ we have

$$
\left|\left\langle u_{+}(z)-u_{-}(\bar{z}), e\right\rangle\right|=2 \varepsilon\left|\left\langle\sqrt{V}(L-\bar{z})^{-1}(L-z)^{-1} u, e\right\rangle\right|=2 \sqrt{\varepsilon}\left|\left\langle(L-z)^{-1} u, D(z) e\right\rangle\right| .
$$

Then, for any compact interval $\Delta \subset \mathbb{R}$ we can write

$$
\begin{aligned}
& \int_{\Delta}\left|\left\langle u_{+}(k+i \varepsilon)-u_{-}(k-i \varepsilon), e\right\rangle\right| d k \\
& \quad \leq 2\left(\int_{\Delta}\|D(k+i \varepsilon) e\|^{2} d k\right)^{1 / 2}\left(\varepsilon \int_{\Delta}\left\|(L-k-i \varepsilon)^{-1} u\right\|^{2} d k\right)^{1 / 2} .
\end{aligned}
$$

The first factor on the right vanishes as $\varepsilon \rightarrow 0$ by assumption and the Lebesgue theorem. We show that the second factor is bounded in $\varepsilon$. An application of the resolvent identity gives

$$
\sqrt{\varepsilon}\left\|(L-z)^{-1} u\right\| \leq \sqrt{\varepsilon}\left\|\left(L^{*}-z\right)^{-1} u\right\|+\|D(z)\| \cdot\left\|\sqrt{V}(L-z)^{-1} u\right\| .
$$


Integrating and taking the inequality (2.3) into account, we find

$$
\varepsilon \int_{\mathbb{R}}\left\|(L-k-i \varepsilon)^{-1} u\right\|^{2} d k \leq 2 \pi\|u\|^{2}+\int_{\mathbb{R}}\left\|\sqrt{V}(L-k-i \varepsilon)^{-1} u\right\|^{2} d k,
$$

which is bounded by the assumption about $u$. We see that the left-hand side of (2.4) converges to 0 , which implies that $\left\langle u_{+}(k)-u_{-}(k), e\right\rangle=0$ a.e. on $\Delta$. Since $\Delta$ and $e$ are arbitrary, we conclude that $u_{+}=u_{-}$.

As has already been mentioned, in the case of a trace class perturbation $V$ a local scattering theory exists for the pair $(L, A)[3$, 4, which implies preservation of the absolutely continuous spectrum as an easy consequence.

Theorem 2.2. If $V \in \mathbf{S}^{1}$, then $\sigma\left(\left.L\right|_{H_{\mathrm{ac}}}\right)=\sigma_{\mathrm{ac}}(A)$, where the absolutely continuous spectrum of the selfadjoint operator $A$ is defined in the standard way.

Since this assertion was not formulated in 3, 4, explicitly, here we give a sketch of its proof.

Proof. By the results in [3, 6], the nuclearity of $V$ implies that any Borel subset $\omega \subset \mathbb{R}$ gives rise to a unique maximal invariant subspace $H_{\omega}$ of the operator $\left.L\right|_{H_{\mathrm{ac}}}$ such that $\sigma\left(\left.L\right|_{H_{\omega}}\right) \subset \bar{\omega}$. This subspace is trivial if $|\omega|=0$, coincides with $H_{\mathrm{ac}}$ for $\omega=\mathbb{R}$, and has the property that if $\omega=\bigcup_{n} \omega_{n}$, then $\sigma\left(\left.L\right|_{H_{\omega}}\right)=\overline{\bigcup_{n} \sigma\left(\left.L\right|_{H_{\omega_{n}}}\right)}$. In the course of the proof of Theorem 5 in [3] it was shown that there exists a scalar contractive analytic function $m$ in the upper half-plane such that for any $c>0$ the bounded local wave operators $W_{ \pm}^{\omega}(A, L): H_{\omega} \rightarrow H$ for the set $\omega=\{k \in \mathbb{R}:|m(k)| \geq c\}$ exist and are complete in the sense that $\operatorname{Ran} W_{ \pm}^{\omega}(A, L)=P_{\omega} H$. Here $P_{\omega}$ is the spectral projection of the a.c. part of $A$ corresponding to the set $\omega$. This means that $\left.L\right|_{H_{\omega}}$ and $A_{\omega}=\left.A\right|_{\operatorname{Ran} P_{\omega}}$ are similar, and thus $\sigma\left(\left.L\right|_{H_{\omega}}\right)=\sigma_{\mathrm{ac}}\left(A_{\omega}\right)$. Now, considering the exhausting sequence $\left\{\omega_{n}\right\}$ of the sets defined as above with $c=1 / n$ and taking into account the fact that the set $\{k: m(k)=0\}$ has Lebesgue measure zero, from the additivity property we conclude that $\sigma\left(\left.L\right|_{H_{\mathrm{ac}}}\right)=\sigma_{\mathrm{ac}}(A)$.

Notice that the statement of the theorem remains true if the weaker condition $(L-z)^{-1}-(A-z)^{-1} \in \mathbf{S}^{1}$ is satisfied. The set $\sigma\left(\left.L\right|_{H_{\mathrm{ac}}}\right)$ is referred to as the absolutely continuous spectrum of the operator $L$.

In general, the question as to whether the condition $L_{1}-L_{2} \in \mathbf{S}^{1}$ for a pair of dissipative operators $L_{1}, L_{2}$ implies $\sigma\left(\left.L_{1}\right|_{H_{\mathrm{ac}}\left(L_{1}\right)}\right)=\sigma\left(\left.L_{2}\right|_{H_{\mathrm{ac}}\left(L_{2}\right)}\right)$ appears to be open if $\operatorname{Im} L_{1,2}$ are not in $\mathbf{S}^{1}$ separately. We shall need a result of this type in a special situation. The original work of this paper starts here.

Lemma 2.3. Let $L$ and $\widetilde{L}$ be dissipative operators such that $\widetilde{L}-L=i \Gamma$ with $a \Gamma \in \mathbf{S}^{1}$. Assume that $\Gamma \geq 0$ and there exists a bounded operator $T \geq 0$ such that $\Gamma=\widetilde{V} T$, $\widetilde{V}=\operatorname{Im} \widetilde{L}$. If $H_{\mathrm{ac}}(\widetilde{L})=\{0\}$, then $H_{\mathrm{ac}}(L)=\{0\}$.

Proof. Let $H_{\mathrm{ac}}(\widetilde{L})=\{0\}$. Applying the criterion of Proposition 2.1 to $\widetilde{L}$ and taking the assumed factorization of $\Gamma$ into account, we see that

$$
\sqrt{\varepsilon}\left(\widetilde{L}^{*}-z\right)^{-1} \sqrt{\Gamma} \stackrel{s}{\longrightarrow} 0
$$

for a.e. $k \in \mathbb{R}$. We show that $\sqrt{\varepsilon}\left(L^{*}-z\right)^{-1} \sqrt{\Gamma} \stackrel{s}{\longrightarrow} 0$ for a.e. $k$. We have

$$
\sqrt{\varepsilon}\left(L^{*}-z\right)^{-1} \sqrt{\Gamma} \cdot G(z)=\sqrt{\varepsilon}\left(\widetilde{L}^{*}-z\right)^{-1} \sqrt{\Gamma},
$$

where

$$
G(z)=I+i \sqrt{\Gamma}\left(\widetilde{L}^{*}-z\right)^{-1} \sqrt{\Gamma} .
$$


Let $V=\operatorname{Im} L$. Then the following calculation shows that $G(z)$ is a contractive function in $\mathbb{C}_{+}$:

$$
\begin{aligned}
& I-G^{*}(z) G(z)=I-\left(I-i \sqrt{\Gamma}(\widetilde{L}-\bar{z})^{-1} \sqrt{\Gamma}\right)\left(I+i \sqrt{\Gamma}\left(\widetilde{L}^{*}-z\right)^{-1} \sqrt{\Gamma}\right) \\
& \quad=i \sqrt{\Gamma}(\widetilde{L}-\bar{z})^{-1}\left(\left(\widetilde{L}^{*}-z\right)-(\widetilde{L}-\bar{z})+i \Gamma\right)\left(\widetilde{L}^{*}-z\right)^{-1} \sqrt{\Gamma} \\
& \quad=\sqrt{\Gamma}(\widetilde{L}-\bar{z})^{-1}(2 \varepsilon+2 V+\Gamma)\left(\widetilde{L}^{*}-z\right)^{-1} \sqrt{\Gamma} \geq 0 .
\end{aligned}
$$

Since $\Gamma$ is of trace class, we have $\sqrt{\Gamma}\left(\widetilde{L}^{*}-z\right)^{-1} \sqrt{\Gamma} \in \mathbf{S}^{1}$. Thus, $G(z)$ is a contractive analytic operator-valued function in $\mathbb{C}_{+}$such that $I-G(z) \in \mathbf{S}^{1}$. Moreover, the operator $G(z)$ is boundedly invertible for $\operatorname{Im} z$ sufficiently large, because $G(z) \rightarrow I$ in the operator norm as $\operatorname{Im} z \rightarrow+\infty$ by the reason that $\widetilde{L}$ has a bounded imaginary part. Hence, by the Szökefalvi-Nagy theorem [5], the function $G(z)$ admits a scalar multiple; that is, there exists a scalar contractive analytic function $g \not \equiv 0$ such that $G(z) \Omega(z)=g(z) \cdot I$ for some bounded analytic function $\Omega$. By the Fatou theorem [5], it follows that the strong boundary values $G^{-1}(k)=\operatorname{s}^{-} \lim _{\varepsilon \downarrow 0} G^{-1}(k+i \varepsilon)$ of the function $G^{-1}$ exist for a.e. $k \in \mathbb{R}$. We infer that

$$
\sqrt{\varepsilon}\left(L^{*}-z\right)^{-1} \sqrt{\Gamma}=\sqrt{\varepsilon}\left(\widetilde{L}^{*}-z\right)^{-1} \sqrt{\Gamma} \cdot G^{-1}(z) \underset{\varepsilon \downarrow 0}{\stackrel{s}{\longrightarrow}} 0
$$

for a.e. $k \in \mathbb{R}$. Now we can verify condition (2.2) for the operator $L$. We have

$$
\sqrt{\varepsilon}\left(L^{*}-z\right)^{-1} \sqrt{V}=\sqrt{\varepsilon}\left(\widetilde{L}^{*}-z\right)^{-1} \sqrt{V}-i \sqrt{\varepsilon}\left(L^{*}-z\right)^{-1} \sqrt{\Gamma} \cdot Q(z),
$$

where $Q(z)=\sqrt{\Gamma}\left(\widetilde{L}^{*}-z\right)^{-1} \sqrt{V}$ is a bounded analytic function in $\mathbb{C}_{+}$. The latter follows from a calculation similar to (2.5), which shows that $I-G(z) G^{*}(z)$ equals the righthand side of (2.5) with $(\widetilde{L}-\bar{z})^{-1}$ and $\left(\widetilde{L}^{*}-z\right)^{-1}$ swapped, and therefore $Q(z) Q^{*}(z) \leq$ $I$. The first term on the right in (2.6) converges strongly to zero by assumption, and an application of the Fatou theorem to the function $Q(z)$ shows that the second term converges strongly to zero as well.

In the next section we establish the triviality of the absolutely continuous subspace for an operator $L$ by verifying condition (2.2) for an operator $\widetilde{L}$ satisfying the assumptions of Lemma 2.3. As the proof of the lemma shows, the final result does not depend then on the "only if" part of Proposition 2.1.

\section{§3. Discrete Schrödinger operator}

Let $\left\{q_{n}\right\}$ be an arbitrary sequence of complex numbers such that $0 \leq \operatorname{Im} q_{n} \leq C$, $C<\infty$. Define $\Theta(z)$ and $\Phi(z), z \in \mathbb{C}$, to be the solutions of the difference equation

$$
u_{n+1}+u_{n-1}+q_{n} u_{n}=z u_{n}
$$

for $n>1$ with the initial conditions $\Theta_{1}=0, \Theta_{2}=-1, \Phi_{1}=1, \Phi_{2}=z-q_{1}$.

The discrete Schrödinger operator $l$ acts in the Hilbert space $H=l^{2}(\mathbb{N})$ and has the form

$$
\begin{gathered}
l=S+S^{*}+Q, \\
(S u)_{n}=u_{n+1}, \quad(Q u)_{n}=q_{n} u_{n} .
\end{gathered}
$$

This formula defines $l$ on the domain of the multiplication operator $Q ; l$ is a maximal dissipative operator, and it is easily seen to be completely nonselfadjoint if $\operatorname{Im} q \not \equiv 0$. For $z \in \mathbb{C}_{-}$, let $u(z)=(l-z)^{-1} \delta_{1}$, where $H \ni \delta_{1}=(1,0, \ldots)$; then $u(z)$ is an $l^{2}$-solution of (3.1) for $n>1$. The function $u$ satisfies

$$
u(z)=-\Theta(z)+m(z) \Phi(z),
$$


where $m(z) \stackrel{\text { def }}{=}\left\langle(l-z)^{-1} \delta_{1}, \delta_{1}\right\rangle$. Obviously, $-m(z)$ is a Nevanlinna function in $\mathbb{C}_{-}$ $(\operatorname{Im} m(z) \leq 0)$, and therefore the finite boundary values $m(k)=\lim _{\varepsilon \downarrow 0} m(k-i \varepsilon)$ exist for almost all $k \in \mathbb{R}$. At such $k$ 's, the solution $u(k), u_{j}(k)=\lim _{\varepsilon \downarrow 0} u_{j}(k-i \varepsilon)$, of (3.1) with $z=k$ is well defined.

Lemma 3.1. If a finite boundary value $m(k)$ exists, then the sum $\sum_{j} \operatorname{Im} q_{j}\left|u_{j}(k)\right|^{2}$ is finite, and

$$
\limsup _{\varepsilon \downarrow 0}\left(\varepsilon\|u(k-i \varepsilon)\|^{2}\right) \leq-\operatorname{Im} m(k) .
$$

Proof. Both assertions result immediately from the identity $(z=k-i \varepsilon, \varepsilon>0)$

$$
\sum_{j=1}^{\infty} \operatorname{Im} q_{j}\left|u_{j}(z)\right|^{2}+\varepsilon \sum_{j=1}^{\infty}\left|u_{j}(z)\right|^{2}=-\operatorname{Im} m(z),
$$

because each term on the left-hand side is nonnegative. To verify this identity, we notice that the left-hand side equals

$$
\operatorname{Im}\langle(l-z) u(z), u(z)\rangle=\operatorname{Im}\left\langle\delta_{1}, u(z)\right\rangle=\operatorname{Im} \overline{m(z)}=-\operatorname{Im} m(z) .
$$

The following lemma provides a convenient condition sufficient for the triviality of the subspace $H_{\mathrm{ac}}(l)$ and stated in terms of solutions of the equation (3.1) for real values of the spectral parameter. For a function $\psi$ analytic in $\mathbb{C}_{-}$, we introduce the notation $\widetilde{\psi}(z)=\overline{\psi(\bar{z})}$. Let $\alpha_{j}=\sqrt{\operatorname{Im} q_{j}}, \alpha=\operatorname{diag}\left\{\alpha_{j}\right\}$, and let $D(z), z=k+i \varepsilon$, be the operator defined in (2.2) with $L=l$.

Lemma 3.2. Let $k \in \mathbb{R}$ be such that $m(k)$ exists finitely. If $\left\{\alpha_{j} \Phi_{j}(k)\right\} \notin l^{2}$, then $D(k+i \varepsilon) \underset{\varepsilon \downarrow 0}{\stackrel{s}{\longrightarrow}} 0$.

Proof. We shall check the required strong convergence on a certain dense set of compactly supported vectors. Let us consider the action of the resolvent of $l^{*}$ on such vectors. A straightforward calculation shows that for any compactly supported $f(z=k+i \varepsilon, \varepsilon>0)$ we have

$$
\left[\left(l^{*}-z\right)^{-1} f\right]_{j}=\widetilde{\Phi}_{j}(z) \sum_{n=j}^{\infty} \widetilde{u}_{n}(z) f_{n}+\widetilde{u}_{j}(z) \sum_{n=1}^{j-1} \widetilde{\Phi}_{n}(z) f_{n} .
$$

The first term on the right vanishes for the $j$ 's to the right of the support of $f$. If $k$ is such that $m(k)$ exists finitely, then $\widetilde{u}_{n}(z)$ converges as $\varepsilon \rightarrow 0$ for each $n$, and both terms on the right-hand side are bounded as $\varepsilon \rightarrow 0$ uniformly in $j \leq N$ for any fixed $N$. Thus, we see that

$$
\begin{aligned}
\left(l^{*}-z\right)^{-1} f & =c_{\varepsilon}[f] \widetilde{u}(z)+r(\varepsilon), \\
c_{\varepsilon}[f] & =\sum_{n=1}^{\infty} \widetilde{\Phi}_{n}(k+i \varepsilon) f_{n}
\end{aligned}
$$

for any $k$ satisfying the assumption of the lemma, where $r(\varepsilon)$ vanishes to the right of the support of $f$ and is bounded uniformly and, moreover, in the $l^{2}$-norm, as $\varepsilon \rightarrow$ 0 . Substituting (3.3) in the definition of $D(z)$ and taking into account the fact that $\varepsilon^{1 / 2}\|\widetilde{u}(z)\|$ is bounded as $\varepsilon \rightarrow 0$ by (3.2), we find that

$$
\|D(z) v\| \leq C\left|c_{\varepsilon}[\alpha v]\right|+o(1), \quad \varepsilon \rightarrow 0,
$$

for any compactly supported $v$. Observe now that the linear set

$$
\mathcal{D}=\left\{v \in l^{2}(\mathbb{N}): v \text { is compactly supported and } \sum_{j} v_{j} \alpha_{j} \widetilde{\Phi}_{j}(k)=0\right\}
$$


is dense in $l^{2}$ if $\left\{\alpha_{j} \Phi_{j}(k)\right\} \notin l^{2}$. Hence, it suffices to check that $D(k+i \varepsilon) v \rightarrow 0$ for $v \in \mathcal{D}$. Indeed, $c_{\varepsilon}[\alpha v] \rightarrow \sum_{n} \alpha_{n} \widetilde{\Phi}_{n}(k) v_{n}=0$ as $\varepsilon \rightarrow 0$ for $v \in \mathcal{D}$, and the required limit relation follows.

Theorem 3.3. Let $\left\{q_{j}\right\}$ be a sequence of complex numbers such that $0 \leq \operatorname{Im} q_{n} \leq C$, $C<\infty$, and $\sigma_{\text {ess }}(l) \subset \mathbb{R}$. If $\operatorname{Im} q \notin l^{1}$, then $H_{\mathrm{ac}}=\{0\}$.

Proof. By the preceding lemma and Proposition 2.1, it suffices to show that if $\operatorname{Im} q \notin l^{1}$, then $\sum_{j} \operatorname{Im} q_{j}\left|\Phi_{j}(k)\right|^{2}=\infty$ for a.e. $k \in \mathbb{R}$. For $k$ such that $m(k)$ exists finitely, consider the Wronskian of the solutions $\Phi$ and $u$,

$$
1=W[\Phi(k), u(k)] \equiv \Phi_{j-1}(k) u_{j}(k)-\Phi_{j}(k) u_{j-1}(k), \quad j>1 .
$$

Multiplying this identity by $\alpha_{j} \alpha_{j-1}$ and summing over $j$, we obtain

$$
\begin{aligned}
\sum^{n} \alpha_{j} \alpha_{j-1} & \leq \sum^{n} \alpha_{j-1}\left|\Phi_{j-1}(k)\right| \cdot \alpha_{j}\left|u_{j}(k)\right|+\sum^{n} \alpha_{j}\left|\Phi_{j}(k)\right| \cdot \alpha_{j-1}\left|u_{j-1}(k)\right| \\
& \leq 2\left(\sum^{n} \operatorname{Im} q_{j}\left|\Phi_{j}(k)\right|^{2}\right)^{1 / 2}\left(\sum^{n} \operatorname{Im} q_{j}\left|u_{j}(k)\right|^{2}\right)^{1 / 2} .
\end{aligned}
$$

By Lemma 3.1, the last factor on the right is bounded in $n$. We see that if $\sum_{j} \alpha_{j} \alpha_{j-1}=$ $\infty$, then $\sum_{j} \operatorname{Im} q_{j}\left|\Phi_{j}(k)\right|^{2}=\infty$ for a.e. $k \in \mathbb{R}$, and the theorem is proved in this case.

The general case reduces to that considered above, because if $\operatorname{Im} q \notin l^{1}$, then there exists a nonnegative $d \in l^{1}$ such that $\widetilde{q}=q+i d$ satisfies $\sum_{j} \widetilde{\alpha}_{j} \widetilde{\alpha}_{j-1}=\infty$ with $\widetilde{\alpha}_{j}=$ $\left(\operatorname{Im} \widetilde{q}_{j}\right)^{1 / 2}$. Indeed, by the uniform boundedness principle, the assumption that $\alpha \notin l^{2}$ implies that there exists $\rho \in l^{2}$ such that $\sum_{j} \rho_{j} \alpha_{j}=\infty$, and, obviously, this $\rho$ can be chosen to be nonnegative. Now we can set $d_{j}=\rho_{j-1}^{2}$, for $\widetilde{\alpha}_{j} \widetilde{\alpha}_{j-1} \geq \rho_{j-1} \alpha_{j-1}$. Application of Lemma 2.3 completes the proof.

Since $\sigma_{\text {ess }}(l) \subset \mathbb{R}$ as $\operatorname{Im} q_{n} \rightarrow 0$, this theorem contains Theorem 1 from the Introduction. Furthermore, in the case where $\operatorname{Im} q \in l^{1}$ the trace class scattering theory implies $\sigma\left(\left.l\right|_{H_{\mathrm{ac}}}\right)=\sigma_{\mathrm{ac}}(\operatorname{Re} l)$, and we see that the theorem gives a complete description of the absolutely continuous spectrum of a dissipative discrete Schrödinger operator.

The above proof extends straightforwardly to Jacobi matrices with selfadjoint offdiagonal part. Namely, given a bounded sequence $\left\{\rho_{n}\right\}$ of positive numbers, we introduce the Jacobi matrix $J$ by

$$
J=\Lambda S+S^{*} \Lambda+Q
$$

where

$$
(\Lambda u)_{n}=\rho_{n} u_{n} .
$$

Then $J$ is a maximal dissipative operator of the class under consideration.

Theorem 3.4. Let $\left\{q_{j}\right\}$ be a sequence of complex numbers such that $0 \leq \operatorname{Im} q_{n} \leq C$, $C<\infty$, and $\sigma_{\mathrm{ess}}(J) \subset \mathbb{R}$. If $\operatorname{Im} q \notin l^{1}$, then $H_{\mathrm{ac}}(J)=\{0\}$.

Indeed, assuming without loss of generality that $\rho_{1}=1$, we can define the solutions $\Theta$ and $\Phi$ of the equation $\rho_{n+1} u_{n+1}+\rho_{n} u_{n-1}+q_{n} u_{n}=z u_{n}$ with the same initial conditions as in the case of $\rho \equiv 1$, and put $u(z)=(J-z)^{-1} \delta_{1}$. The proof then proceeds verbatim as in the case of $\rho \equiv 1$ until (3.4), where we shall have

$$
W_{j}[\Phi, u] \equiv \Phi_{j-1}(k) u_{j}(k)-\Phi_{j}(k) u_{j-1}(k)=\rho_{j-1}^{-1}
$$

instead. Obviously, the left-hand side is bounded away from zero, since the $\rho_{j}$ are bounded, and the rest of the proof coincides with that of Theorem 3.3 , 


\section{$\S 4$. Continuous Case}

1. Preliminaries and the beginning of the proof of the main result. Let $q$ be a locally bounded function on $\mathbb{R}_{+}$satisfying $\operatorname{Im} q(x) \geq 0$ a.e., and let $h$ be an arbitrary real number. We shall assume throughout that $\operatorname{Im} q \in L^{\infty}\left(\mathbb{R}_{+}\right)$and that $\operatorname{Re} q$ is bounded below. Define $\Theta(x, z)$ and $\Phi(x, z)$ to be the solutions to

$$
-u^{\prime \prime}+\bar{q} u=z u, \quad z \in \mathbb{C},
$$

with the Cauchy data $\Phi(0, z)=\Theta^{\prime}(0, z)=0, \Phi^{\prime}(0, z)=\Theta(0, z)=1$. It is standard that the solutions $\Theta$ and $\Phi$ are continuous in $z$ uniformly in $x \in I$ for any compact interval $I$.

The Schrödinger operator $l, l y=-y^{\prime \prime}+q y$, is defined in the Hilbert space $H=L^{2}\left(\mathbb{R}_{+}\right)$ on the domain of its real part, the Schrödinger operator with the potential $\operatorname{Re} q$, fixed by the selfadjoint boundary condition $y^{\prime}(0)=h y(0)$. Under the assumptions imposed, $l$ is a completely nonselfadjoint maximal dissipative operator, provided that $\operatorname{Im} q \not \equiv 0[8$.

The following lemma is a continuous analog of Lemma 3.1.

Lemma 4.1. For any $z \in \mathbb{C}_{+}$there exists exactly one linearly independent $L^{2}$-solution $u(x, z)$ of (4.1), which can be chosen in the form

$$
u(x, z)=\Theta(x, z)+m(z) \Phi(x, z),
$$

where $m$ is an analytic function in $\mathbb{C}_{+}$. For almost all $k \in \mathbb{R}$ the function $m$ has finite boundary values, $m(k)=\lim _{\varepsilon \downarrow 0} m(k+i \varepsilon)$, and the solution $u(x, k)=\lim _{\varepsilon \downarrow 0} u(x, k+i \varepsilon)$ satisfies

$$
\int \operatorname{Im} q(x)|u(x, k)|^{2} d x<\infty .
$$

Moreover, for any $\varepsilon>0$ we have

$$
\operatorname{Im} m(z)=\int_{0}^{\infty}(\operatorname{Im} q(t)+\varepsilon)|u(t, z)|^{2} d t, \quad z=k+i \varepsilon .
$$

In particular,

$$
\limsup _{\varepsilon \downarrow 0}\left(\varepsilon\|u(\cdot, k+i \varepsilon)\|^{2}\right) \leq \operatorname{Im} m(k)
$$

whenever $m(k)$ exists.

Proof. As is well known (see, e.g., [13]), the assumption that $\operatorname{Re} q$ is bounded below implies that the differential expression corresponding to the potential $\operatorname{Re} q$ is in the limit-point case at infinity; that is, the minimal operator $\widetilde{l}=\left.\operatorname{Re} l\right|_{C_{0}^{\infty}}$ has deficiency indices $(1,1)$. It follows that the operator $l_{0}=\widetilde{l}+i \operatorname{Im} q$ satisfies $\operatorname{dim} \operatorname{ker}\left(l_{0}^{*}-z\right)=1$ for $\operatorname{Im} z$ sufficiently large, and therefore for all $z \in \mathbb{C}_{+}$, because $\mathbb{C}_{-} \subset \widehat{\rho}\left(l_{0}\right)$. Thus, (4.1) has exactly one linearly independent $L^{2}$-solution for all $z \in \mathbb{C}_{+}$.

Assume now that the solution is representable as in (4.2).$^{2}$ Since $\operatorname{Re} q$ is bounded below, the identity

$$
0=\int_{0}^{x}\left(-u^{\prime \prime}+(\bar{q}-z) u\right) \bar{u} d t=-u^{\prime}(x) \overline{u(x)}+m(z)+\int_{0}^{x}(\bar{q}-z)|u|^{2} d t+\int_{0}^{x}\left|u^{\prime}\right|^{2} d t
$$

shows that the function $\left|u^{\prime} \bar{u}\right|$ has a limit, finite or infinite, as $x \rightarrow+\infty$. Taking the real part, we find

$$
\frac{1}{2} \frac{d}{d x}|u(x)|^{2}=\text { const }+\int_{0}^{x} \operatorname{Re}(q-z)|u|^{2} d t+\int_{0}^{x}\left|u^{\prime}\right|^{2} d t
$$

\footnotetext{
${ }^{2}$ Once the uniqueness of an $L^{2}$-solution is established, the representation (4.2) and identity (4.4) are results of the nesting circles analysis of equation (4.1) carried out in 11. For completeness, here we provide a simple derivation of these facts, except for the analyticity of $m$.
} 
which implies that $u^{\prime} \in L^{2}$ (otherwise $|u(x)| \rightarrow \infty$ as $x \rightarrow+\infty$ ). This shows that $u^{\prime}(x) \overline{u(x)} \rightarrow 0$ as $x \rightarrow+\infty$. Taking the imaginary part of (4.6) and passing to the limit as $x \rightarrow+\infty$, we obtain (4.4). This shows that $m(z)$ is a Nevanlinna function in $\mathbb{C}_{+}$ $(\operatorname{Im} m(z) \geq 0)$ and therefore has finite boundary values for a.e. $k$ on the real axis, and for any such $k$ the integral $\int{ }^{x} \operatorname{Im} q(t)|u(t, k)|^{2} d t$ is bounded in $x$ (by $\operatorname{Im} m(k)$ ). It follows that the integral in (4.3) is finite.

To establish the representation (4.2), it suffices to observe that $\Phi(x, z)$ cannot be an $L^{2}$-solution for $z \in \mathbb{C}_{+}$, for otherwise a calculation similar to the above would lead to the formula $\int_{0}^{\infty}(\operatorname{Im} q+\varepsilon)|\Phi|^{2} d t=0$ implying that $\Phi \equiv 0$. The analyticity of the function $m$ was established in [11].

We shall often suppress the argument $x$ in the notation and write $u(z)$ for the element of the space $H$ determined by the function $u(x, z)$ for each $z \in \mathbb{C}_{+}$.

From now on, we shall assume that $\operatorname{Im} q(x) \rightarrow 0$ as $x \rightarrow \infty$, which guarantees that $\sigma_{\text {ess }}(l) \subset \mathbb{R}$ by a relative compactness argument. Then $l$ is an operator of the class considered in $\S 2$.

In the following lemma, $D(z)$ is defined by (2.2) with $L=l$. Let $u_{h}=\Theta+h \Phi$.

Lemma 4.2. Let $k \in \mathbb{R}$ be such that the finite boundary value $m(k)$ exists, and let $m(k) \neq h$. Then

(i) for any compactly supported $v \in H(z=k+i \varepsilon)$ we have

$$
D(z) v=\sqrt{\varepsilon} C_{\varepsilon}[v] u(z)+o(1), \quad \varepsilon \rightarrow 0,
$$

where

$$
C_{\varepsilon}[v]=\frac{1}{h-m(k+i \varepsilon)} \int_{0}^{\infty} u_{h}(x, k+i \varepsilon) \sqrt{\operatorname{Im} q(x)} v(x) d x,
$$

and the o-symbol refers to the $L^{2}$-norm;

(ii) if $\sqrt{\operatorname{Im} q} u_{h}(\cdot, k) \notin L^{2}$, then $D(k+i \varepsilon) \underset{\varepsilon \downarrow 0}{\stackrel{s}{\longrightarrow}} 0$.

Proof. The arguments are completely similar to the proof of Lemma 3.2. Let $f \in H$ be supported on a finite interval $[0, X]$. It is straightforward to check that

$$
\begin{aligned}
& \left(\left(l^{*}-z\right)^{-1} f\right)(x) \\
& \quad=\frac{1}{h-m(z)}\left[u(x, z) \int_{0}^{x} u_{h}(s, z) f(s) d s+u_{h}(x, z) \int_{x}^{\infty} u(s, z) f(s) d s\right]
\end{aligned}
$$

for all $z \in \mathbb{C}_{+}$. Obviously, the function in the square brackets, which will be denoted by $g$, coincides with

$$
\left(\int_{0}^{\infty} u_{h}(s, z) f(s) d s\right) u(x, z)
$$

for $x>X$. Moreover, $g(x, z)$ converges as $\varepsilon \rightarrow 0$ uniformly in $x \in[0, X]$. Since the condition $m(k) \neq h$ secures the boundedness of the common factor in (4.9), this gives

$$
\left(l^{*}-z\right)^{-1} f=\left(\frac{1}{h-m(z)} \int_{0}^{\infty} u_{h}(s, z) f(s) d s\right) u(z)+r(z),
$$

where the function $r(x, z)$ vanishes for $x>X$ and is uniformly bounded as $\varepsilon \rightarrow 0$. This implies (i).

Notice that, by (4.5), $\varepsilon^{1 / 2}\|u(k+i \varepsilon)\|$ is bounded as $\varepsilon \rightarrow 0$, so that $\|D(z) v\| \leq$ $C\left|C_{\varepsilon}[v]\right|+o(1)$ for any compactly supported $v$. Now, define $C_{0}[v]$ by formula (4.8) with $\varepsilon=0$. If $v$ satisfies $C_{0}[v]=0$, then $C_{\varepsilon} \rightarrow 0$, and thus $D(z) v \rightarrow 0$. Since the set of compactly supported $v$ such that $C_{0}[v]=0$ is dense in $L^{2}$ if $\sqrt{\operatorname{Im} q} u_{h}(\cdot, k) \notin L^{2}$, statement (ii) follows. 
Since the set of all $k$ such that $m(k)=h$ has measure zero by the uniqueness theorem for Nevanlinna functions, we have the following.

Corollary 4.3. If $\sqrt{\operatorname{Im} q} u_{h}(\cdot, k) \notin L^{2}$ for a.e. $k \in \mathbb{R}$, then $H_{\mathrm{ac}}(L)=\{0\}$.

Before proving the main result (Theorem 2), we would like to give a motivation of it in terms of the WKB asymptotics for the solutions. As is shown in the asymptotic theory of ordinary differential equations [12, for potentials $q$ behaving at infinity in a sufficiently regular way the asymptotics of solutions of equation (4.1) as $x \rightarrow \infty$ can be calculated explicitly. In particular, the theory asserts the existence of two solutions $u_{ \pm}$ of the equation $-u^{\prime \prime}+\bar{q} u=k u$ for $k>0$ with the asymptotics of the form

$$
u_{ \pm} \sim \exp \left[ \pm i\left(\sqrt{k} x-\frac{1}{2 \sqrt{k}} \int^{x} \overline{q(\xi)} d \xi\right)\right], \quad x \rightarrow \infty
$$

(the WKB asymptotics) if $q$ belongs to certain classes of decaying functions. For instance, the asymptotics (4.10) occurs for all $k>0$ if $q \in L^{2}$ and $q^{\prime} \in L^{1}$ [12. Let $\operatorname{Im} q \notin L^{1}$.

Observe that then the solution $u_{-}$grows, $\left|u_{-}\right| \sim \exp \left(\frac{1}{2 \sqrt{k}} \int^{x} \operatorname{Im} q d \xi\right)$, while $u_{+}$decays. It should be noted that if the WKB asymptotics occurs for a given $k>0$, and a solution $v$ of (4.1) with $z=k>0$ satisfies $\sqrt{\operatorname{Im} q} v \in L^{2}$, then $v$ must be a multiple of $u_{+}$, because

$$
\begin{aligned}
\int^{\infty} & \operatorname{Im} q\left|u_{-}\right|^{2} d x \\
& \sim \int^{\infty} \operatorname{Im} q(x) \exp \left(C \int^{x} \operatorname{Im} q(\xi) d \xi\right) d x=\exp \left(C \int^{\infty} \operatorname{Im} q(x) d x\right)=\infty .
\end{aligned}
$$

A decaying function $q$ will be called a WKB-class potential if the asymptotics (4.10) occurs for a.e. $k>0$. It follows that, if $q$ is a WKB-class potential, then the solutions $u_{h}(\cdot, k)$ and $u(\cdot, k)$ must coincide for a.e. $k>0$ such that $\sqrt{\operatorname{Im} q} u_{h}(\cdot, k) \in L^{2}$. This is only possible on the set of $k$ such that $m(k)=h$; hence $\sqrt{\operatorname{Im} q} u_{h}(\cdot, k) \notin L^{2}$ for a.e. $k>0$. Applying Corollary 4.3, we thus obtain Theorem 2 for WKB-class potentials. In particular, by a result quoted above, this shows immediately that the operator with the potential $q(x)=i x^{-\beta}, 1 / 2<\beta \leq 1$, has trivial absolutely continuous subspace. Notice that the eigenvalues of this operator only accumulate at 0 by a complex scaling argument (a remark due to S. Naboko).

Now, we proceed to the proof of Theorem 2. The argument becomes less direct than in the discrete case because estimating the Wronskian of solutions $u$ and $u_{h}$ requires a bound on their derivatives, which is only available in the mean with respect to the spectral parameter and leads to some mild conditions on the real part of the potential in the theorem. The proof involves spectral averaging for the selfadjoint problem corresponding to the potential $\operatorname{Re} q[14$ ] and the Gilbert-Pearson subordinacy theory [13. The required part of the Last-Simon result reads as follows.

Theorem 4.4 ([14, Theorem $2.1 \mathrm{C}]$ ). Let $V$ be a real potential locally bounded and bounded below, and let $d \mu=\min \left\{d \rho^{D}, d \rho^{N}\right\}$, where the $d \rho^{D / N}$ are the spectral measures for the selfadjoint Schrödinger operators with potential $V$ and Dirichlet-Neumann boundary conditions at zero, respectively. Let $\Theta$ and $\Phi$ be the solutions to $-y^{\prime \prime}+V y=k y$ with the Cauchy data $\Phi(0, k)=\Theta^{\prime}(0, k)=0, \Phi^{\prime}(0, k)=\Theta(0, k)=1$. Then for any compact interval $I \subset \mathbb{R}$ there exists a constant $C(I)$ such that for all $x>0$ we have

$$
\begin{array}{r}
\int_{I}\left(|\Theta(x, k)|^{2}+|\Phi(x, k)|^{2}\right) d \mu(k) \leq C(I), \\
\int_{I}\left[\int_{x-1}^{x+1}\left(\left|\Theta^{\prime}(y, k)\right|^{2}+\left|\Phi^{\prime}(y, k)\right|^{2}\right) d y\right] d \mu(k) \leq C(I) .
\end{array}
$$


If additionally $\sup _{n} \int_{n}^{n+1}|V(t)|^{2} d t$ is finite, then

$$
\int_{I}\left(\left|\Theta^{\prime}(x, k)\right|^{2}+\left|\Phi^{\prime}(x, k)\right|^{2}\right) d \mu(k) \leq C(I) .
$$

In terms of the transfer matrix $T(x, k)$ for the differential equation $-y^{\prime \prime}+V y=k y$, (4.11) and (4.13) together are equivalent to saying that

$$
\int_{I}\|T(t, k)\|^{2} d \mu(k) \leq C(I)
$$

We shall need the following lemma of some interest on its own.

Lemma 4.5. Let $y_{0}$ be any solution of the equation $-y^{\prime \prime}+\bar{q} y=k y$ such that $r=$ $y_{0}^{\prime}(0) / y_{0}(0) \in \mathbb{R}\left(\right.$ or $\left.y_{0}(0)=0\right)$. Then for any solution $y$ of this equation there exists a constant $C$ such that $|y(x)| \leq C\left|y_{0}(x)\right|$ for $x$ sufficiently large, provided that $\operatorname{Im} q \not \equiv 0$.

Proof. As we shall see later, the solution $y_{0}(x)$ does not vanish for large $x .^{3}$ Therefore, it suffices to establish the required estimate for the solution

$$
y_{1}(x)=\left(\int^{x} \frac{d \xi}{y_{0}^{2}(\xi)}\right) y_{0}(x)
$$

linearly independent of $y_{0}$. Multiplying the equation for $y_{0}$ by $\overline{y_{0}}$ and integrating from 0 to $x$, we obtain

$$
-\left.y_{0}^{\prime}(x) \overline{y_{0}(x)}\right|_{0} ^{x}+\int_{0}^{x}\left|y_{0}^{\prime}\right|^{2} d t+\int_{0}^{x}(\bar{q}-k)\left|y_{0}\right|^{2} d t=0 .
$$

Taking the imaginary part and observing that $y_{0}^{\prime}(0) \overline{y_{0}(0)}=r\left|y_{0}(0)\right|^{2}$ is real, we find

$$
-\operatorname{Im}\left[y_{0}^{\prime}(x) \overline{y_{0}(x)}\right]=\int_{0}^{x} \operatorname{Im} q\left|y_{0}\right|^{2} d t .
$$

We denote the left-hand side by $\rho(x)$. The above identity shows that $\rho(x)>0$ for large $x$, whence $y_{0}(x) \neq 0$, and we can introduce variables $R, \Psi(R>0)$ by $y_{0}=R e^{-i \Psi}$. By direct inspection, we have $\rho=R^{2} \Psi^{\prime}$, so that $\Psi^{\prime}>0$, and the phase $\Psi$ can be chosen to be a continuous monotone increasing function. Then we have

$$
\int^{x} \frac{d \xi}{y_{0}^{2}(\xi)}=\int^{x} e^{2 i \Psi(\xi)} \frac{\Psi^{\prime}(\xi) d \xi}{R^{2}(\xi) \Psi^{\prime}(\xi)}=\int^{\Psi(x)} \frac{e^{2 i \Psi} d \Psi}{\rho(\xi(\Psi))} .
$$

The nonnegative function $\zeta(s)=1 / \rho(\xi(s))$ is monotone decreasing, which implies that the integral on the right is uniformly bounded, and the result follows.

This lemma shows, in particular, that the solution $u_{h}(x, k)$ estimates the solution $u(x, k)$ from above whenever the latter exists, $u=O\left(\left|u_{h}\right|\right)$ at large $x$. In the following lemma we use standard asymptotic perturbation theory [12] to compare the asymptotics of solutions corresponding to the potentials $q$ and $\operatorname{Re} q$.

Lemma 4.6. Let $k \in \mathbb{R}$ be such that $m(k)$ exists, and let $m(k) \neq h$. If $\sqrt{\operatorname{Im} q} u_{h}(\cdot, k) \in$ $L^{2}$, then the equation

$$
-y^{\prime \prime}+\operatorname{Re} q y=k y
$$

has solutions $y_{1,2}$ such that $y_{1}=u_{h}(1+o(1)), y_{2}=u+o\left(\left|u_{h}\right|\right)$ as $x \rightarrow \infty$, and the Wronskian $W\left[y_{1}, y_{2}\right]$ satisfies

$$
W\left[y_{1}, y_{2}\right]=W\left[u, u_{h}\right]=m(k)-h .
$$

\footnotetext{
${ }^{3}$ This can also be seen immediately from the fact that a completely nonselfadjoint dissipative operator has no real eigenvalues.
} 
Proof. Rewriting equation (4.15) in the matrix form,

$$
Y^{\prime}=\left(\begin{array}{cc}
0 & 1 \\
\operatorname{Re} q-k & 0
\end{array}\right) Y, \quad Y=\left(\begin{array}{ll}
y_{1} & y_{2} \\
y_{1}^{\prime} & y_{2}^{\prime}
\end{array}\right)
$$

and resorting to variation of the parameter formula, we obtain the equation

$$
Z^{\prime}=\frac{i}{h-m(k)}\left(\begin{array}{cc}
\operatorname{Im} q u_{h} u & \operatorname{Im} q u^{2} \\
-\operatorname{Im} q u_{h}^{2} & -\operatorname{Im} q u_{h} u
\end{array}\right) Z
$$

where

$$
Y=\left(\begin{array}{cc}
u_{h} & u \\
u_{h}^{\prime} & u^{\prime}
\end{array}\right) Z
$$

The fact that $\sqrt{\operatorname{Im} q} u(\cdot, k) \in L^{2}$ (see (4.3) $)$ and the assumption $\sqrt{\operatorname{Im} q} u_{h}(\cdot, k) \in L^{2}$ imply that

$$
\left(\begin{array}{cc}
\operatorname{Im} q u_{h} u & \operatorname{Im} q u^{2} \\
-\operatorname{Im} q u_{h}^{2} & -\operatorname{Im} q u_{h} u
\end{array}\right) \in L^{1} .
$$

Applying now the Levinson theorem to equation (4.17), we see that it has a solution $Z$ satisfying $Z(x)=I+o(1)$. Since $u=O\left(\left|u_{h}\right|\right)$, we obtain the existence of the solutions $y_{1,2}$. Calculating the determinant of $Y$ at infinity, we obtain (4.16).

Proof of Theorem 2 (beginning). Suppose that $H_{\mathrm{ac}}$ is nontrivial. Then, by Proposition 2.1. there exists a set $M \subset \sigma_{\text {ess }}(l)$ of positive Lebesgue measure such that $D(k+i \varepsilon)$ does not converge to 0 strongly as $\varepsilon \downarrow 0$ for $k \in M$, and therefore the function $\sqrt{\operatorname{Im} q} u_{h}(\cdot, k)$ belongs to $L^{2}$ for a.e. $k \in M$ by Lemma 4.2 (ii). For such $k$ 's, let $y_{1,2}(\cdot, k)$ be the solutions of (4.15) described ${ }^{4}$ in Lemma 4.6. From the lemma it is immediate that the solutions $y_{1,2}(\cdot, k)$ are square integrable with the weight $\operatorname{Im} q$ for a.e. $k \in M$. Let $\mu$ be the a.c. measure given by $d \mu=\min \left\{d \rho^{D}, d \rho^{N}\right\}$, where the $d \rho^{D / N}$ are the spectral measures for the selfadjoint Schrödinger operators with potential $\operatorname{Re} q$ and DirichletNeumann boundary conditions at zero, respectively. We fix arbitrary $c_{0}, N$, and $A$ such that the set

$$
I=\left\{k \in M:|m(k)| \leq c_{0}, \int_{0}^{\infty} \operatorname{Im} q\left(\left|y_{1}(t, k)\right|^{2}+\left|y_{2}(t, k)\right|^{2}\right) d t \leq N\right\} \cap[-A, A]
$$

has positive $\mu$-measure. This is possible because, as we shall show later, $\mu(M)>0$. Let $\rho(k)=1+\max \left\{\left|y_{1,2}(0, k)\right|,\left|y_{1,2}^{\prime}(0, k)\right|\right\}$, and let $T(x, k)$ be the transfer matrix for equation (4.15). Then $\left|y_{1,2}^{\prime}(x, k)\right| \leq \rho(k)\|T(x, k)\|$. Taking (4.16) into account, we have

$$
|h-m(k)|=\left|W\left[y_{1}, y_{2}\right]\right| \leq \rho(k)\|T(x, k)\|\left(\left|y_{1}\right|+y_{2} \mid\right) .
$$

Multiplying this by $(\rho(k))^{-1} \operatorname{Im} q$ and integrating, we obtain

$$
\begin{aligned}
& \int_{I} \frac{|h-m(k)|}{\rho(k)} d \mu(k) \int^{x} \operatorname{Im} q d t \\
& \quad \leq C\left(\int_{I} d \mu(k) \int^{x} \operatorname{Im} q\left(\left|y_{1}\right|^{2}+\left|y_{2}\right|^{2}\right) d t\right)^{1 / 2}\left(\int^{x} d t \operatorname{Im} q(t) \int_{I}\|T(t, k)\|^{2} d \mu(k)\right)^{1 / 2} .
\end{aligned}
$$

Now, $\int_{I}\|T(t, k)\|^{2} d \mu(k)$ is bounded uniformly in $t$ by Theorem 4.4 (see (4.14)). Here we have used the assumption that $\operatorname{Re} q$ is uniformly locally $L^{2}$-bounded. This gives

$$
C\left(\int^{x} \operatorname{Im} q d t\right)^{1 / 2} \leq C\left(\int_{I} d \mu(k) \int^{x} \operatorname{Im} q\left(\left|y_{1}\right|^{2}+\left|y_{2}\right|^{2}\right) d t\right)^{1 / 2} \leq C \sqrt{N \mu(I)}
$$

and so the integral on the left is bounded.

${ }^{4}$ Without loss of generality, we may assume that $m(k)$ exists and $m(k) \neq h$ for all $k \in M$. 
Thus, it remains to show that $\mu(M)>0$. This will occupy the rest of the paper and requires developing an analog of the subordinacy theory [13] for dissipative Schrödinger operators.

2. Subordinacy theory and the end of the proof of Theorem 2. Recall [13] that a solution $u_{1}$ of a second-order differential equation on the semiaxis $[0, \infty)$ is called subordinate if

$$
\frac{\left\|u_{1}\right\|_{N}}{\left\|u_{2}\right\|_{N}} \rightarrow 0
$$

as $N \rightarrow \infty$ for any solution $u_{2}$ linearly independent of $u_{1}$. Here $\|\cdot\|_{N} \equiv\|\cdot\|_{L^{2}(0, N)}$.

Theorem 4.7. Let $\operatorname{Im} q \not \equiv 0$. Then for a.e. $k \in \mathbb{R}$ the following assertions are equivalent.

(i) $D(k+i \varepsilon) \underset{\varepsilon \downarrow 0}{\stackrel{s}{\longrightarrow}} 0$.

(ii) There exists a subordinate solution to $-y^{\prime \prime}+q y=k y$.

As the proof shows, the set of full measure in this theorem can be identified with the set of real $k$ such that $m(k)$ exists finitely and $m(k) \neq h$. We shall provide a complete proof of the implication $(i i) \Longrightarrow(i)$ only, because the reverse implication is not used in this paper. The proof is subdivided into several assertions.

Lemma 4.8. For any real $k$ such that $m(k)$ exists and $m(k) \neq h$, assertion (i) $i s$ equivalent to saying that

$$
\varepsilon\|u(\cdot, k+i \varepsilon)\|^{2} \rightarrow 0 .
$$

Proof. Lemma 4.2(i) shows that for any compactly supported $v \in H$ we have

$$
\|D(k+i \varepsilon) v\| \leq C \varepsilon^{1 / 2}\|u(k+i \varepsilon)\|+o(1),
$$

because $C_{\varepsilon}[v]$ given by (4.8) converges as $\varepsilon \rightarrow 0$. This implies that if (4.19) is satisfied, then $D(k+i \varepsilon) v \rightarrow 0$ for any compactly supported $v \in H$, and (i) follows.

To establish the reverse implication, we choose $v$ to be $\overline{u_{h}(x, k)} \chi(x)$ where $\chi$ is the indicator of an interval $\Delta$. Then

$$
C_{\varepsilon}[v] \underset{\varepsilon \rightarrow 0}{\longrightarrow} \frac{1}{h-m(k)} \int_{\Delta}\left|u_{h}(s, k)\right|^{2} \sqrt{\operatorname{Im} q} d s \neq 0
$$

if $\Delta$ is sufficiently large. Now, Lemma 4.2(i) shows that if $D(z) v \rightarrow 0$, then (4.19) is true.

The proof of the following assertion, which is central in the subordinacy theory, carries over, with a slight simplification, from the selfadjoint theory [13].

Proposition 4.9. If the equation $-y^{\prime \prime}+\bar{q} y=k y$ has a subordinate solution for some $k \in \mathbb{R}$ such that $m(k)$ exists and $m(k) \neq h$, then this solution is a multiple of $u(x, k)$.

Proof. Without loss of generality, we may assume that $u(\cdot, k) \notin L^{2}$. Let $v$ be the subordinate solution in question, with the normalization chosen so that $v=\Theta+c_{0} \Phi$, so that the Wronskian $W[\Phi, v]$ is equal to 1 . This is possible since, by Lemma 4.5, $\Phi$ cannot be a subordinate solution. We must prove that $c_{0}=m(k)$.

A straightforward calculation shows that the solution $u(x, z)$ satisfies the following integral equation:

$$
g(x)=\Theta(x, k)+m(z) \Phi(x, k)-i \varepsilon\left[\Phi(x, k) \int_{0}^{x} v(s) g(s) d s-v(x) \int_{0}^{x} \Phi(s, k) g(s) d s\right] .
$$

We consider this equation in $L^{2}(0, A(\varepsilon))$ with a finite $A(\varepsilon)$ to be chosen so that $A(\varepsilon) \rightarrow \infty$ as $\varepsilon \rightarrow 0$. If

$$
\varepsilon\|\Phi(\cdot, k)\|_{A(\varepsilon)}\|v\|_{A(\varepsilon)}<\frac{1}{2}
$$


then the equation can be iterated in $L^{2}(0, A(\varepsilon))$ to give

$$
\begin{aligned}
& \|u(\cdot, z)\|_{A(\varepsilon)} \asymp\|\Theta(\cdot, k)+m(z) \Phi(\cdot, k)\|_{A(\varepsilon)}=\left\|v(\cdot, k)+\left(m(z)-c_{0}\right) \Phi(\cdot, k)\right\|_{A(\varepsilon)} \\
& \quad=\left(\left|m(z)-c_{0}\right|+o(1)\right)\|\Phi(\cdot, k)\|_{A(\varepsilon)},
\end{aligned}
$$

where we have used the fact that $v$ is subordinate, and therefore

$$
\|v\|_{A(\varepsilon)} /\|\Phi(\cdot, k)\|_{A(\varepsilon)} \rightarrow 0 .
$$

We define $A(\varepsilon)$ by

$$
\begin{gathered}
\|\Phi(\cdot, k)\|_{A(\varepsilon)}\|v\|_{A(\varepsilon)}=\frac{1}{3 C}\|u(\cdot, k+i \varepsilon)\|^{2}, \\
C=\limsup _{\varepsilon \rightarrow 0} \operatorname{Im} m(k+i \varepsilon) .
\end{gathered}
$$

Then (4.20) is satisfied by (4.5), and $A(\varepsilon)$ tends to infinity as $\varepsilon \downarrow 0$ because otherwise $\|u(\cdot, k)\| \leq \liminf \|u(\cdot, k+i \varepsilon)\|$ would be finite. Thus, we obtain

$$
\left|c_{0}-m(z)\right| \leq \frac{\|u(\cdot, k+i \varepsilon)\|}{\|\Phi(\cdot, k)\|_{A(\varepsilon)}}+o(1)=\left(3 C \frac{\|v\|_{A(\varepsilon)}}{\|\Phi(\cdot, k)\|_{A(\varepsilon)}}\right)^{1 / 2}+o(1) \rightarrow 0 .
$$

Proof of Theorem 4.7. (ii) $\Longrightarrow$ (i) To establish this implication it remains to show that if $u(\cdot, k)$ is a subordinate solution, then (4.19) is satisfied. We shall actually show that if $u(\cdot, k)$ is subordinate, then, moreover,

$$
\int_{0}^{\infty} \operatorname{Im} q(x)|u(x, k)|^{2} d x=\operatorname{Im} m(k) .
$$

From (4.4) it is clear that condition (4.19) then follows. We shall use an argument from the proof of Lemma 4.5. Multiplying the identity $-u^{\prime \prime}+(\bar{q}-k) u=0$ by $\bar{u}$, integrating and taking the imaginary part, we obtain

$$
\operatorname{Im}\left[u^{\prime}(x, k) \overline{u(x, k)}\right]=\operatorname{Im} m(k)-\int_{0}^{x} \operatorname{Im} q(t)|u(t, k)|^{2} d t .
$$

By (4.4), the right-hand side is nonnegative for all $x$, and so the required assertion is immediate if the left-hand side has a zero. Suppose now that the left-hand side does not vanish for all $x>0$, so that, at least, $u(x) \neq 0$, and we can introduce variables $R, \Psi(R>0)$ by $u=R e^{i \Psi}$. Then the left-hand side becomes $\rho(x):=R^{2}(x) \Psi^{\prime}(x)$. Since $\rho(x)$ is positive, it follows that the phase $\Psi$ can be chosen to be a continuous monotone increasing function.

Then, for a solution $v$ linearly independent of $u$, we have

$$
\begin{aligned}
v(x) & =\left(\int_{a}^{x} \frac{d \xi}{u^{2}(\xi)}\right) u(x) \\
& =\left(\int^{x} e^{-2 i \Psi(\xi)} \frac{\Psi^{\prime}(\xi) d \xi}{R^{2}(\xi) \Psi^{\prime}(\xi)}\right) u(x)=\left(\int^{\Psi(x)} e^{-2 i \Psi} \zeta(\Psi) d \Psi\right) u(x),
\end{aligned}
$$

where $\zeta(s)=1 / \rho\left(\Psi^{-1}(s)\right)$ is a positive monotone increasing function. It follows that $\zeta(s) \rightarrow \infty$; otherwise the integral would be bounded and $v$ would be bounded above by $u$, which contradicts the assumption that $u$ is subordinate. Thus, $\int_{0}^{x} \operatorname{Im} q|u|^{2} d t \rightarrow \operatorname{Im} m(k)$ as $x \rightarrow \infty$, as required.

Remark 1 . In the selfadjoint theory the assertion corresponding to the last part of the proof of Theorem 4.7 (if $u(\cdot, k)$ is a subordinate solution, then $\operatorname{Im} m(k)=0$ ) is trivial, because a subordinate solution normalized by the condition $u(0)=1$ must be real when $\operatorname{Im} q \equiv 0$. 
Remark 2. We have left unproved only one assertion in the implication $(i) \Longrightarrow(i i)$ of Theorem 4.7 if $\varepsilon\|u(k+i \varepsilon)\|^{2} \rightarrow 0$ for some $k \in \mathbb{R}$ such that $m(k)$ exists and $m(k) \neq h$, then $u(x, k)$ is a subordinate solution. The proof of this fact can be taken verbatim from the corresponding assertion in the selfadjoint theory [13].

Proof of Theorem 2 (end). We must show that $\mu(M)>0$. Suppose, by contradiction, that $\mu(M)=0$. Since $\mu$ is equivalent to the a.c. part of the spectral measure of the selfadjoint Schrödinger operator with the potential Re $q$, the following assertion of the selfadjoint Gilbert-Pearson theory applies.

Proposition (see [13]). Let $V$ be a locally bounded real potential in the limit point case at infinity, and let $d \nu$ be the a.c. part of the spectral measure for the selfadjoint Schrödinger operator with the potential $V$ and a selfadjoint boundary condition at zero. If $\nu(S)=0$, then the equation $-y^{\prime \prime}+V y=k y$ has a subordinate solution for Lebesgue-almost-all $k \in S$.

This shows that equation (4.15) has a subordinate solution for a.e. $k \in M$. Lemma 4.6 now implies that the equation $-y^{\prime \prime}+\bar{q} y=k y$ has a subordinate solution for a.e. $k \in M$ as well. Indeed, let $y=c_{0} y_{1}+y_{2}$ be a subordinate solution of (4.15). Then $y \sim u+c_{0} u_{h}+o\left(u_{h}\right)$, whence

$$
\frac{\left\|u+c_{0} u_{h}\right\|_{N}}{\left\|u_{h}\right\|_{N}} \sim \frac{\|y\|_{N}}{\left\|y_{1}\right\|_{N}} \rightarrow 0
$$

that is, $u+c_{0} u_{h}$ is a subordinate solution of (4.1) with $z=k$. Since $y_{1}$ cannot be a subordinate solution, we are done. Applying Theorem 4.7, we obtain $D(k+i \varepsilon) \stackrel{s}{\longrightarrow} 0$ for a.e. $k \in M$, a contradiction.

As was mentioned above, conditions on the real part of the potential in Theorem 2 are required to apply spectral averaging. In particular, the condition that $\operatorname{Re} q$ is uniformly locally $L^{2}$-bounded allows us to use the following standard estimate for derivatives of solutions of a second-order differential equation (see, for instance, [15, Lemma 3.1]): if $\sup _{n} \int_{n}^{n+1}|V(t)|^{2} d t$ is finite, then

$$
\left|g^{\prime}(x)\right|^{2} \leq C\left(1+k^{2}\right) \int_{x-1}^{x+1}|g(s)|^{2} d s
$$

for any solution $g$ to $-y^{\prime \prime}+V y=k y$ with a constant $C$ depending on the potential $V$ only. As was noticed in [14, a combination of this bound with (4.11) allows us to drop the additional integration in $y$ in (4.12) and to arrive at (4.13). By the following theorem, if we omit the condition on the positive part of $\operatorname{Re} q$, then the nontriviality of $H_{\mathrm{ac}}$ implies that $\operatorname{Im} q$ is in $L^{1}$ in an averaged sense.

Theorem 4.10. Let $q$ be a locally bounded function on $\mathbb{R}_{+}$with $\operatorname{Im} q \geq 0, \operatorname{Im} q(x) \rightarrow 0$ as $x \rightarrow \infty$. Assume that $\operatorname{Re} q$ is bounded below. If the absolutely continuous subspace of the Schrödinger operator $l$ is nontrivial, then

$$
\sum_{n}\left(\int_{n}^{n+1} \sqrt{\operatorname{Im} q} d t\right)^{2}<\infty
$$

Proof. The argument develops as before until the Wronskian estimate (4.18), which we multiply by $(\rho(k))^{-1} \sqrt{\operatorname{Im} q}$ and integrate in $t$ from $n$ to $n+1$ and in $k$, to obtain

$$
\int_{I} \frac{|h-m(k)|}{\rho(k)} d \mu(k) \int_{n}^{n+1} \sqrt{\operatorname{Im} q} d t \leq C\left(\int_{I} d \mu(k) \int_{n}^{n+1} \operatorname{Im} q\left(\left|y_{1}\right|^{2}+\left|y_{2}\right|^{2}\right) d t\right)^{1 / 2} .
$$


Here we have taken (4.12) into account. Squaring and summing over $n$, we arrive at the result.

It remains unclear if it is possible to carry the proof of Theorem 2 over to the discrete case. The difficulty here is that no appropriate discrete analog of the trigonometric substitution $u=R e^{i \Psi}$ in the proof of Theorem 4.7 is known.

Finally, it should be mentioned that the condition of pointwise decay of $\operatorname{Im} q$ as $x \rightarrow \infty$ in Theorem 2 can be replaced by any other condition guaranteeing that $\sigma_{\text {ess }}(l) \subset \mathbb{R}$.

\section{$\S 5$. Concluding Remarks}

As is well known [5], the triviality of the subspace $H_{\mathrm{ac}}$ implies that the characteristic function of the operator is inner. So far, the analysis of operators with inner characteristic functions has mainly been developed for the discrete part of the spectrum and requires the existence of a scalar multiple [16], thus rendering it efficient in the case of trace class perturbations only. Examples considered in this paper suggest that a nontrivial "singular" inner factor of the characteristic function appears naturally in applications when the perturbation is not of trace class.

It should also be mentioned that the instability phenomenon described in this paper is likely to be a specific feature of dissipative Schrödinger operators. In the nondissipative case, a WKB-class potential will have all solutions of (4.1) bounded for a.e. $z>0$ if its imaginary part is conditionally integrable. This suggests that, in this case, the absolutely continuous subspace can be nontrivial even if $\operatorname{Im} q \notin L^{1}$.

Acknowledgements. The author is indebted to S. Naboko for helpful and encouraging discussions and to M. Marletta for noticing a mistake in the initial formulation of Lemma 4.6 .

\section{REFERENCES}

[1] P. Deift and R. Killip, On the absolutely continuous spectrum of one-dimensional Schrödinger operators with square summable potentials, Comm. Math. Phys. 203 (1999), 341-347. MR1697600 (2000i:34223)

[2] M. Christ and A. Kiselev, Scattering and wave operators for one-dimensional Schrödinger operators with slowly decaying nonsmooth potentials, Geom. Funct. Anal. 12 (2002), 1174-1234. MR1952927 (2003m:47019)

[3] S. N. Naboko, Functional model of perturbation theory and its applications to scattering theory, Trudy Mat. Inst. Steklov. 147 (1980), 86-114; English transl. in Proc. Steklov Inst. Math. 1981, no. 2. MR0573902 (82d:47012)

[4] - On the conditions for existence of wave operators in the nonselfadjoint case, Wave Propagation. Scattering Theory, Probl. Mat. Fiz., vyp. 12, Leningrad. Univ., Leningrad, 1987, pp. 132-155; English transl., Amer. Math. Soc. Transl. Ser. 2, vol. 157, Amer. Math. Soc., Providence, RI, 1993, pp. 127-149. MR0923975 (90b:47019)

[5] B. Sz.-Nagy and C. Foiaş, Analyse harmonique des opérateurs de l'espace de Hilbert, Masson et Cie, Paris; Akad. Kiadó, Budapest, 1967. MR0225183 (37:778)

[6] B. S. Pavlov, Expansion in eigenfunctions of the absolutely continuous spectrum of a dissipative operator, Vestnik Leningrad. Univ. Mat. Mekh. Astronom. 1975, vyp. 1, 130-137; English transl. in Vestnik Leningrad Univ. Math. 8 (1980). MR0425657 (54:13611)

[7] L. A. Sakhnovich, Dissipative operators with absolutely continuous spectrum, Trudy Moskov. Mat. Obshch. 19 (1968), 211-270; English transl., Trans. Moscow Math. Soc. 19 (1968), 233-297. MR0250109(40:3350)

[8] B. S. Pavlov, Selfadjoint dilation of a dissipative Schrödinger operator, and expansion in its eigenfunction, Mat. Sb. (N.S.) 102 (144) (1977), no. 4, 511-536; English transl., Math. USSR-Sb. 31 (1977), no. 4, 457-478. MR0510053 (58:23154)

[9] A. S. Tikhonov, Absolutely continuous spectrum of a linear operator and problems of scattering theory and of factorization of operator-functions, Ph. D. thesis, Simferopol. Gos. Univ., Simferopol, 1989. (Russian) 
[10] R. Romanov, A remark on equivalence of weak and strong definitions of the absolutely continuous subspace for nonself-adjoint operators, Oper. Theory Adv. Appl., vol. 154, Birkhäuser, Basel, 2004, pp. 179-184. MR2103375 (2005g:47064)

[11] A. R. Sims, Secondary conditions for linear differential operators of the second order, J. Math. Mech. 6 (1957), no. 2, 247-285. MR0086224(19:144g)

[12] M. S. P. Eastham, The asymptotic solution of linear differential systems, London Math. Soc. Monogr. New Ser., vol. 4, The Clarendon Press, Oxford Univ. Press, New York, 1989. MR1006434 (91d:34001)

[13] D. B. Pearson, Quantum scattering and spectral theory, Techn. Phys., vol. 9, Acad. Press, London, 1988. MR 1099604 (91k:81198)

[14] Y. Last and B. Simon, Eigenfunctions, transfer matrices, and absolutely continuous spectrum of one-dimensional Schrödinger operators, Invent. Math. 135 (1999), no. 2, 329-367. MR1666767 (2000f:47060)

[15] B. Simon, Bounded eigenfunctions and absolutely continuous spectra for one-dimensional Schrödinger operators, Proc. Amer. Math. Soc. 124 (1996), 3361-3369. MR.1350963 (97a:34223)

[16] N. K. Nikol'skiŭ, Treatise of the shift operator. Spectral functionl theory, "Nauka", Moscow, 1980; English transl., Grundlehren Math. Wiss., vol. 273, Springer-Verlag, Berlin, 1986. MR0827223 (87i:47042).

School of Computer Science, Cardiff University, Cardiff, Queen's Buildings, PO Box 916, Newport Road, Cardiff CF24 3XF, United Kingdom, and Laboratory of Quantum Networks, Institute for Physics, St. Petersburg State University, St. Petersburg 198504, Russia

E-mail address: roma@rvr.stud.pu.ru

Received 14/JAN/2004

Translated by THE AUTHOR 